\title{
Efficacy and safety of PICOPREP tailored dosing compared with PICOPREP day-before dosing for colon cleansing: a multi-centric randomised study
}

\section{(이요 $\odot$}

\author{
Authors \\ Ralf Kiesslich ${ }^{1}$, Stefan Schubert ${ }^{2}$, Michael Mross ${ }^{3}$, Tobias Klugmann ${ }^{4}$, \\ Michael Klemt-Kropp ${ }^{5}$, Imke Behnken ${ }^{6}$, Gillaume Bonnaud ${ }^{7}$, Eric \\ Keulen ${ }^{8}$, Marcel Groenen ${ }^{9}$, Michael Blaker ${ }^{10}$, Thierry Ponchon ${ }^{11}$, \\ Wilfred Landry ${ }^{12}$, Meredin Stoltenberg ${ }^{13}$ \\ Institutions \\ 1 Klinikdirektor ZIM II, Sprecher ZIM (Zentrum für Innere \\ Medizin), Helios Dr. Horst Schmidt Kliniken Wiesbaden, \\ Germany \\ 2 Gastroenterologie am Bayerischen Platz, Berlin, Germany \\ 3 Praxis Dr. med. Michael R Mross, Berlin, Germany \\ 4 Internistische Gemeinschaftspraxis, Leipzig, Germany \\ 5 Medisch Centrum, Alkmaar, the Netherlands \\ 6 Internistisches Facharztzentrum Dr M Scholz, Langen, Germany \\ 7 Clinique des Cèdres, Blagnac, France \\ 8 Orbis Medisch Centrum, Sittard, the Netherlands \\ 9 Rijnstate Arnhem, Wagnerlaan, the Netherlands \\ 10 Gastroenterologie Eppendorfer Baum, Hamburg, Germany \\ 11 Ed Herriot Hopital, Lyon, France \\ 12 MVZ Dachau, Dachau, Germany \\ 13 Ferring Pharmaceuticals, Kay Fiskers Plads 11 Copenhagen S, \\ Denmark
}

submitted 4.4.2016

accepted after revision 19.1.2017

Bibliography

DOI http://dx.doi.org/10.1055/s-0043-102433 |

Endoscopy International Open 2017; 05: E282-E290

(c) Georg Thieme Verlag KG Stuttgart · New York

ISSN 2364-3722
Corresponding author

Prof. Dr. med. Ralf Kiesslich, Klinik für Innere Medizin II (ZIM II),

Schwerpunkte: Gastroenterologie, Hepatologie, Endokrinologie

und Pneumologie, HELIOS Dr. Horst Schmidt Kliniken Wiesbaden,

Ludwig-Erhard-Straße 100, 65199 Wiesbaden

Fax: 0611-432418

ralf.kiesslich@helios-kliniken.de

\section{ABSTRACT}

Background and study aims The success of any colonoscopy procedure depends upon the quality of bowel preparation. We evaluated the efficacy and safety of a new tailored dosing (TD) regimen compared with the approved PICOPREP day-before dosing regimen (DBD) in the European Union.

Patient and methods Patients ( $\geq 18$ years) undergoing colonoscopy were randomised (2:1) to TD (Dose 1, 10-18 hours; Dose 2, 46 hours before colonoscopy) or DBD (Dose 1 before 8:00AM on the day before colonoscopy; Dose 2, 6- 8 hours after Dose 1). The primary endpoint of overall colon cleansing efficacy was based on total Ottawa Scale (OS) scores ( $0-14$, excellent-worst). The key secondary endpoint was a binary endpoint based on the ascending colon OS (success 0 or 1 , failure [ $\geq 2]$ ). Convenience and satisfaction were evaluated similar to the primary and key secondary endpoints. Safety and tolerability were also evaluated.

Results Use of the PICOPREP TD regimen resulted in a statistically significant reduction in the mean total Ottawa Scale score compared to the PICOPREP DBD regimen $(-3.93,95 \%$ confidence intervals $[C I]:-4.99,-2.97 ; P<0.0001)$ in the intent-to-treat analysis set. The PICOPREP TD regimen also resulted in a statistically significant increase in the odds of achieving an ascending colon OS score $\leq 1$, compared to the PICOPREP DBD regimen (estimated odds ratio $9.18,95 \% \mathrm{Cl}: 4.36,19.32 ; P<0.0001)$. There was no statistically significant difference in the overall rate of treatment-emergent adverse events (12\% (TD) and $5.7 \%$ (DBD), respectively, $P=0.2988$ ). The convenience and satisfaction were comparable in the two groups.

Conclusion The TD regimen was superior to the DBD regimen for overall and ascending colon cleansing efficacy.

ClinicalTrials.gov Identifier: NCT02239692

\section{Introduction}

Colonoscopy procedures are now being routinely used for evaluating the colon, mostly for screening and early detection of colorectal cancer, which has a high incidence and mortality [1]. Extensive data show that adequate bowel preparation is instrumental in success of any colonoscopy procedure [2]. Poor bowel preparation accounts for nearly $20 \%$ of failed colonoscopies, limiting the procedure's diagnostic/therapeutic value [2].
Also, patients may require a repeat colonoscopy, adding to the burden for both patients and colonoscopists, and increased costs [2,3].

Of various factors governing the quality of bowel preparation, concerns such as unpleasant taste of the preparation and/or cumbersome experience of taking large volumes of liquid have been addressed to a great extent [4-6]. However, guidelines [7,8] and emerging data $[9,10]$ suggest that dosing regimen, and importantly, the time between the last dose of 
bowel preparation and the colonoscopy procedure are instrumental in achieving a successful colonoscopy. Hence, there have been continued efforts to improve bowel cleansing by optimizing the dosing regimen [9-11].

Day-before dosing (DBD) regimens may be an option for (in particular) morning colonoscopies [4], however, they introduce a time interval of at least 12 hours between the last dose and an afternoon colonoscopy procedure. During that time, chyme from the small intestine accumulates and coats the proximal colon, hindering visualization and detection of flat lesions [9, $11,12]$. To address this, split-dosing regimens have been introduced $[9,11,13]$. This reduces the time interval between the last dose and afternoon/evening colonoscopy procedure and is associated with improved quality of bowel preparation $[14,15]$. The split-dosing regimen of PICOPREP has been shown to be efficacious, safe and well-tolerated [13,16-21], and has also been studied in children [22-24].

The current non-inferiority study evaluated efficacy and safety of a new tailored dosing (TD) regimen, which is customised based on time of colonoscopy, and also offers ease of consumption similar to split-dosing vs. the approved DBD regimen of PICOPREP for bowel preparation prior to colonoscopy in the European Union.

\section{Patients and methods}

\section{Patients}

Patients aged $\geq 18$ years who were scheduled to undergo an elective colonoscopy were enrolled. Patients had to have $\geq 3$ spontaneous bowel movements/week for a month prior to inclusion in the study. Furthermore, patients were excluded from the study if they had acute surgical abdominal conditions, active inflammatory bowel disease, colon disease (toxic megacolon, toxic colitis, idiopathic pseudo-obstruction, and hypomotility syndrome), ascites, gastrointestinal disorders (active ulcers, gastric outlet obstruction, retention, gastroparesis, and ileus), uncontrolled angina and/or myocardial infarction (within the past 3 months), congestive heart failure or uncontrolled hypertension, severely reduced renal function (glomerular filtration rate $<30 \mathrm{~mL} / \mathrm{min} / 1.73 \mathrm{~m}^{2}$ ), rhabdomyolysis, nausea, vomiting and hypermagnesaemia, and severe dehydration at screening. Patients who had a previous gastrointestinal surgery (including gastric resection, banding, or bypass), those participating in other studies, and those who were hypersensitive to the study drug were also excluded. Pregnant and lactating women, and patients in any way related to study or site staff were not eligible to participate.

Concomitant use of lithium, laxatives, constipating drugs, antidiarrheal agents, or oral iron preparations were not permitted in the study, and it was ensured that their use was suspended before administration of the bowel preparation.

\section{Study design and treatment}

This was a Phase 3, randomized, assessor-blinded, multicenter study comparing efficacy and safety of the PICOPREP TD regimen to the DBD regimen for bowel preparation prior to colonoscopy. Randomization was stratified by time of colonoscopy procedure, i. e. morning or afternoon, in a 2:1 ratio to either the PICOPREP TD regimen or to the PICOPREP DBD regimen. Patients were followed up until 7 days after the procedure.

PICOPREP (sodium picosulfate $10 \mathrm{mg}$; magnesium oxide $3.5 \mathrm{~g}$, and citric acid $12 \mathrm{~g}$ ) powder for oral solution was provided in sachets and was administered in 2 divided doses. PICOPREP was reconstituted by mixing the contents of the sachet in a cup with approximately $150 \mathrm{~mL}$ of cold water. Patients were instructed to consume at least $5250-\mathrm{mL}$ glasses of clear liquids, spread over several hours, after the first administration of PICOPREP and at least $3250-\mathrm{mL}$ glasses of clear liquids, spread over several hours, after the second administration. For the TD regimen, the first dose was administered 10 to 18 hours before the colonoscopy, and the second dose was administered 4 to 6 hours before the colonoscopy. For the DBD regimen, the first dose was taken the day before colonoscopy before $8 \mathrm{am}$, and the second dose was taken 6 to 8 hours later, also on the day before colonoscopy, in accordance with the approved EU label.

Patient compliance was monitored by recording the date and quantity of PICOPREP dispensed and used by each patient, volume of liquid intake, time between intake of each dose and actual start time of the colonoscopy corresponding to the $2 \mathrm{PI}$ COPREP dosing regimens. Patients were contacted by phone on the last working day before bowel preparation administration and were reminded of the time of the administrations.

The study is registered at ClinicalTrials.gov (NCT02239692). The patients were recruited from 11 sites across Germany, France and the Netherlands between November 2014 and June 2015. The study was approved by an independent ethics committee for all participating sites, and was conducted in accordance with the Declaration of Helsinki, Good Clinical Practice, and applicable regulatory requirements. All patients provided written informed consent.

\section{Study endpoints and assessments}

The primary endpoint was the total Ottawa Scale score, derived from a validated 5 -grade Ottawa Scale ( $\triangleright$ Table 1 ). The total Ottawa Scale score is calculated by adding the ratings ( 0 to 4 ) for each of the 3 colon segments and the overall fluid quantity rating (0 to 2 ) and ranges from 0 (best) to 14 (worst) for the overall assessment of colon cleansing. The key secondary endpoint was a binary endpoint based on whether the ascending colon Ottawa Scale score $\leq 1$. The other efficacy endpoints were mid (transverse, descending) and recto-sigmoid colon cleansing, assessed depending on whether the Ottawa Scale score $\leq 1$ for the corresponding colon segment. The proportion of repeat colonoscopy procedures was also evaluated. Convenience and satisfaction of both dosing regimens, absence from work and impact on daily activities were assessed by separate patient questionnaires (Appendix), on the day of colonoscopy, prior to sedation (if needed). Safety was assessed throughout the study by recording adverse events (AEs), findings on physical examinations, orthostatic vital sign measurements, laboratory test results (haematology, coagulation, blood chemistry, and urinalysis), and 12-lead electrocardiograms (ECGs). The AEs were coded using Medical Dictionary for Regulatory Activities, version 17.0 . 


\begin{tabular}{|c|c|}
\hline Score & Description \\
\hline 0 & $\begin{array}{l}\text { Excellent: Mucosal detail clearly visible. If fluid is present, } \\
\text { it is clear. Almost no stool residue. }\end{array}$ \\
\hline 1 & $\begin{array}{l}\text { Good: Some turbid fluid or stool residue but mucosal } \\
\text { detail still visible. Washing and suction not necessary. }\end{array}$ \\
\hline 2 & $\begin{array}{l}\text { Fair: Turbid fluid or stool residue obscuring mucosal detail. } \\
\text { However, mucosal detail becomes visible with suctioning. } \\
\text { Washing not necessary. }\end{array}$ \\
\hline 3 & $\begin{array}{l}\text { Poor: Presence of stool obscuring mucosal detail and con- } \\
\text { tour. However, with suctioning and washing, a reasonable } \\
\text { view is obtained. }\end{array}$ \\
\hline 4 & $\begin{array}{l}\text { Inadequate: Solid stool obscuring mucosal detail and con- } \\
\text { tour despite aggressive washing and suctioning. }\end{array}$ \\
\hline
\end{tabular}

\section{Statistical assumptions and analysis}

The sample size calculation was based on the assumptions of an overall significance level $\alpha$ of 0.05 , a power (1- $\beta$ ) of 0.85 , a noninferiority margin of 1.5 units, a standard deviation (SD) of 3.2 for the total Ottawa Scale score, and a dropout rate of $5 \%$ for randomized patients. With these assumptions, a total of 198 patients were needed to be randomized in a 2:1 ratio to either the TD regimen or to the $\mathrm{DBD}$ regimen.

The intention-to-treat (ITT) population consisted of all randomized patients. For patients in the ITT analysis set with partially or completely missing Ottawa Scale scores, the missing values were imputed using the worst possible score. The perprotocol (PP) population consisted of all patients in the ITT population with no major protocol deviations deemed to impact the efficacy of the PICOPREP dosing regimens. The completer analysis set consisted of all patients in the ITT population who underwent the colonoscopy and for whom, complete Ottawa Scale scores were available. The completer analysis set was used to perform sensitivity analyses assessing the impact of the imputation algorithm on the primary analysis.

In order to control the family wise error rate for the primary and key secondary endpoints, the following hierarchical testing procedure was pre-specified in the trial protocol:

1. Test non-inferiority of the PICOPREP TD regimen compared to the DBD regimen with respect to the primary endpoint at a $5 \%$ significance level.

2. Test the superiority of the PICOPREP TD regimen vs. the DBD regimen with respect to the key secondary endpoint at a $5 \%$ significance level.

3. Test the superiority of the PICOPREP TD regimen vs. DBD regimen with respect to the primary endpoint at a $5 \%$ significance level.

Regulatory guidelines require non-inferiority to be established in the both the ITT and PP populations [25]. This requirement is driven by the fact that analyses based on the ITT principle are not necessarily deemed to be conservative in the non-inferiority setting. Therefore, the primary analysis was performed

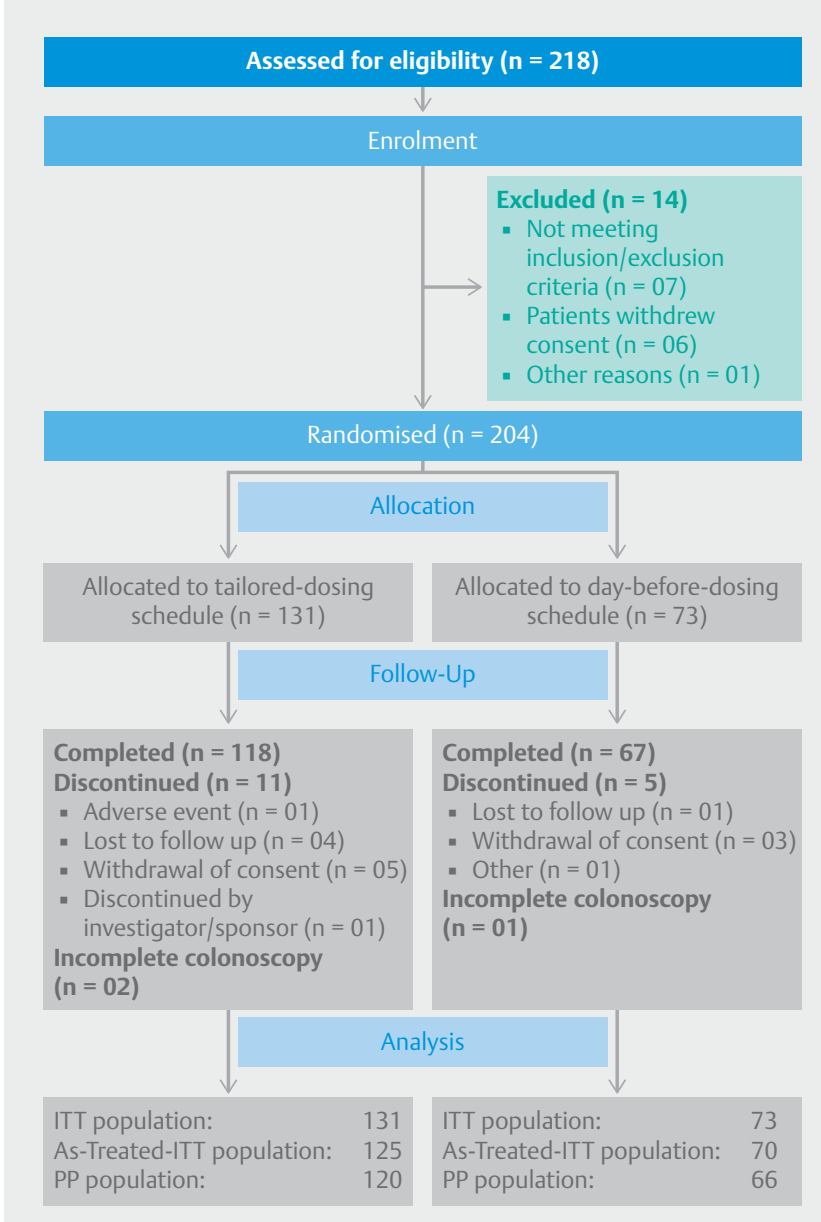

- Fig. 1 CONSORT flow diagram.

As-Treated-ITT analysis set comprised of patients in the ITT analysis set who received the planned treatment, and excluded were the patients who were not treated.

ITT, intention-to-treat; PP, per-protocol

for both the ITT and PP populations while other efficacy analyses were performed only in the ITT population.

The primary analysis compared the difference in the mean total Ottawa Scale score between the PICOPREP TD and DBD regimens based on an analysis of covariance (ANCOVA) controlling for time of colonoscopy (AM/PM) and country. The analysis of the secondary efficacy endpoints was based on logistic regression, controlling for time of colonoscopy (AM/PM) and country.

Convenience, satisfaction, absence from work (duration) and impact on daily activities were evaluated similar to the primary and key secondary endpoints.

\section{Results}

\section{Patient demographics and baseline characteristics}

A total of 204 patients were randomized in the study and 195 patients $(95.6 \%)$ received the study drug. Patient disposition and analysis populations are presented in $>$ Fig. 1. Baseline patient characteristics are presented in $\mathbf{D}$ Table $\mathbf{2}$. 
- Table 2 Demographic and baseline characteristics.

\begin{tabular}{|c|c|c|}
\hline & $\begin{array}{l}\text { Tailored dosing } \\
\text { (N=131) }\end{array}$ & $\begin{array}{l}\text { Day-before dosing } \\
\text { (N=73) }\end{array}$ \\
\hline - Mean (SD) & $58.4(13.3)$ & $56.6(15.1)$ \\
\hline - $\mathrm{n}(\%)$ & $54(41.2): 77(58.8)$ & $30(41.1): 43(58.9)$ \\
\hline Ethnicity, n (\%) & & \\
\hline - Hispanic & $3(2.3)$ & $2(2.7)$ \\
\hline - Not Hispanic & $128(97.7)$ & $71(97.3)$ \\
\hline Race, n (\%) & & \\
\hline - Asian & $1(0.8)$ & - \\
\hline - White & $130(99.2)$ & $73(100.0)$ \\
\hline BMI (kg/m $\left.{ }^{2}\right)$ & & \\
\hline - Mean (SD) & $26.5(4.71)$ & $25.6(4.33)$ \\
\hline
\end{tabular}

\section{Overall colon cleansing efficacy based on Ottawa Scale scores}

Estimated differences in mean total Ottawa Scale score between the TD and DBD regimens for the ITT and PP populations were $-3.93(95 \% \mathrm{Cl}:[-4.99,-2.87])$ and $-4.38(95 \% \mathrm{Cl}$ : $[-5.34,-3.41])$. This clearly establishes non-inferiority, as well as superiority of the TD regimen vs. DBD regimen, with respect to overall colon cleansing efficacy as illustrated in - Fig. 2.

A sensitivity analysis, based on the completer analysis set was performed to assess the impact of the imputation methods on the primary analysis. The results of this completer analysis are also presented in $\boldsymbol{\nabla}$ Fig. $\mathbf{2}$. The consistency of these results suggest that the imputation method did not impact the conclusions of the study.

The observed distribution of Ottawa Scale scores, summarized by colon segment and dosing schedule, are provided in

- Table 3. The observed proportion of patients reporting Otta- wa Scale segment scores of either 0 (excellent) or 1 (good), in - Table 3, further demonstrates the improved efficacy of the TD regimen vs. the DBD regimen.

\section{Ascending, mid and recto-sigmoid colon cleansing efficacy}

The estimated odds of the Ottawa Scale score being $\leq 1$ for the ascending, mid and recto-sigmoid colon segments are presented in > Fig. 3, along with the associated odds ratios comparing the cleansing efficacy of the TD and DBD regimens in each coIon segment. The estimated odds ratios, 9.18 (95\% Cl: 4.36, 19.32; $P<0.0001), 6.85$ (95\% Cl: 3.48, 13.48; $P<0.0001)$ and 6.73 (95\% Cl: $3.53,12.85 ; P<0.0001)$ indicate a statistically significant improvement in colon cleansing efficacy of the TD regimen compared to the DBD regimen in the ascending, mid and recto-sigmoid colon segments, respectively.

\section{Convenience, satisfaction and overall experience}

Results of the Convenience and Satisfaction Questionnaire are presented in $>$ Table 4 . Ease of consuming the study drug was similar for both dosing regimens and was rated as easy to very easy by $93.6 \%$ and $95.6 \%$ of subjects in the TD and DBD arms, respectively. Convenience and satisfaction were rated as good or excellent by $88 \%$ and $85.7 \%$ of the patients following the TD and DBD regimen, respectively. Of the patients who were randomized to the TD regimen and responded to the questionnaire, $97.6 \%$ stated they would request PICOPREP, if they required a colonoscopy in the future. The difference in the adjusted mean number of hours missed from work due to the colon cleansing procedure was negligible between the dosing regimens $(-0.87$ (95\% Cl:-3.47, 1.72; $P=0.5056)$. The adjusted difference in the mean score for the impact question (Did the colon cleansing treatment affect your regular daily activities, other than work at a job?) was significantly lower in the TD regimen vs. DBD regimen $(-0.84,95 \% \mathrm{Cl}:-1.52,-0.17 ; \quad P=$ $0.0147)$.

\begin{tabular}{|ccc|c|}
\hline Analysis set & Treatment & Adjusted mean & Treatment contrast \\
\cline { 2 - 3 } ITT & TD & 4.26 & -3.93 \\
\cline { 2 - 3 } & DBD & 8.19 & \\
\hline \multirow{2}{*}{ Per-Protocol } & TD & 3.70 & -4.38 \\
\cline { 2 - 3 } & DBD & 8.08 & \\
\hline Completer & TD & 3.64 & -4.21 \\
\cline { 2 - 3 } & DBD & 7.85 & \\
\hline
\end{tabular}

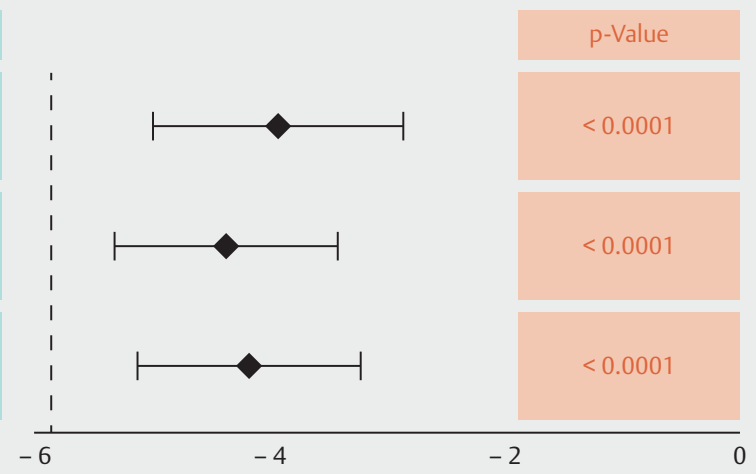

Fig. 2 Difference in total Ottawa Scale scores between the tailored dosing and the day-before dosing regimens (ITT, PP and completer analysis set).

Lower Ottawa Scale scores correspond to better colon cleansing efficacy.

DBD, day-before dosing; ITT, intention-to-treat; PP, per-protocol; TD, tailored dosing 
- Table 3 Observed total Ottawa Scale score distribution by colon segment and dosing schedule (ITT population).

\begin{tabular}{|c|c|c|}
\hline & Tailored dosing $(\mathrm{N}=131)$ & Day-before dosing $(\mathrm{N}=73)$ \\
\hline Ascending colon (numerical), Mean (SD) & $1.4(1.14)$ & $2.8(1.08)$ \\
\hline \multicolumn{3}{|l|}{ Ascending colon (categorical), \% (r/n) } \\
\hline - Score 0 & $23.7(31 / 131)$ & $1.4(1 / 73)$ \\
\hline - Score 1 & $37.4(49 / 131)$ & $13.7(10 / 73)$ \\
\hline - Score 2 & $25.2(33 / 131)$ & $19.2(14 / 73)$ \\
\hline - Score 3 & $6.1(8 / 131)$ & $32.9(24 / 73)$ \\
\hline - Score 4 & $7.6(10 / 131)$ & $32.9(24 / 73)$ \\
\hline Mid colon (numerical), Mean (SD) & $1.2(1.20)$ & $2.4(1.25)$ \\
\hline \multicolumn{3}{|l|}{ Mid colon (categorical), \% (r/n) } \\
\hline - Score 0 & $37.4(49 / 131)$ & $9.6(7 / 73)$ \\
\hline - Score 1 & $27.5(36 / 131)$ & $12.3(9 / 73)$ \\
\hline - Score 2 & $23.7(31 / 131)$ & $32.9(24 / 73)$ \\
\hline - Score 3 & $3.8(5 / 131)$ & $20.5(15 / 73)$ \\
\hline - Score 4 & $7.6(10 / 131)$ & $24.7(18 / 73)$ \\
\hline Recto-sigmoid colon (numerical), Mean (SD) & $1.0(1.15)$ & $2.1(1.27)$ \\
\hline \multicolumn{3}{|l|}{ Recto-sigmoid colon (categorical), \% (r/n) } \\
\hline - Score 0 & $42.0(55 / 131)$ & $13.7(10 / 73)$ \\
\hline - Score 1 & $32.1(42 / 131)$ & $16.4(12 / 73)$ \\
\hline - Score 2 & $13.7(18 / 131)$ & $30.1(22 / 73)$ \\
\hline - Score 3 & $6.9(9 / 131)$ & $23.3(17 / 73)$ \\
\hline - Score 4 & $5.3(7 / 131)$ & $16.4(12 / 73)$ \\
\hline The global fluid quantity rating (numerical), Mean (SD) & $0.7(0.73)$ & $0.9(0.73)$ \\
\hline \multicolumn{3}{|l|}{ The global fluid quantity rating (Categorical), \% (r/n) } \\
\hline - Score 0 & $47.3(62 / 131)$ & $31.5(23 / 73)$ \\
\hline - Score 1 & $36.6(48 / 131)$ & $46.6(34 / 73)$ \\
\hline - Score 2 & $16.0(21 / 131)$ & $21.9(16 / 73)$ \\
\hline
\end{tabular}

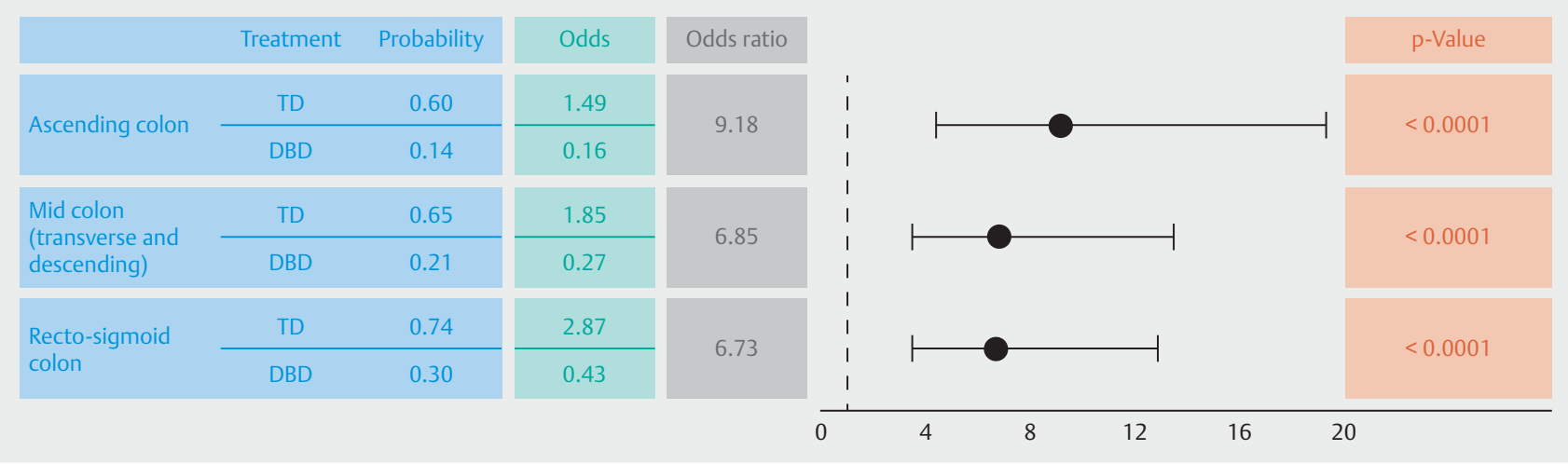

- Fig. 3 Responder status of tailored dosing and day-before dosing regimens (ITT population).

DBD, day-before dosing; ITT, intention-to-treat; TD, tailored dosing 
Table4 Summary of the reported subject convenience and satisfaction - intention-to-treat analysis set.

\begin{tabular}{|c|c|c|}
\hline & Tailored dosing $(\mathbf{N}=131)$ & Day-before dosing $(\mathrm{N}=73)$ \\
\hline & $\%(r / n)$ & $\%(r / n)$ \\
\hline \multicolumn{3}{|c|}{ 1: How easy or difficult was it to consume the PICOPREP medication? } \\
\hline Very easy & $66.4(83 / 125)$ & $72.9(51 / 70)$ \\
\hline Easy & $27.2(34 / 125)$ & $22.9(16 / 70)$ \\
\hline Tolerable & $6.4(8 / 125)$ & $4.3(3 / 70)$ \\
\hline \multicolumn{3}{|l|}{ Difficult } \\
\hline \multicolumn{3}{|c|}{ Very difficult } \\
\hline \multicolumn{3}{|c|}{ 2: Were you able to consume the entire PICOPREP medication as instructed? } \\
\hline Yes & $100.0(125 / 125)$ & $100.0(70 / 70)$ \\
\hline \multicolumn{3}{|l|}{ No } \\
\hline \multicolumn{3}{|c|}{ 3: Please describe your overall experience with the PICOPREP medication. } \\
\hline Excellent & $28.0(35 / 125)$ & $17.1(12 / 70)$ \\
\hline Good & $60.0(75 / 125)$ & $68.6(48 / 70)$ \\
\hline Fair & $12.0(15 / 125)$ & $12.9(9 / 70)$ \\
\hline Poor & & $1.4(1 / 70)$ \\
\hline \multicolumn{3}{|l|}{ Bad } \\
\hline \multicolumn{3}{|c|}{ 5: Would you ask your doctor for this colon cleansing medication if you need another colonoscopy in the future? } \\
\hline Yes & $97.6(122 / 125)$ & $92.9(65 / 70)$ \\
\hline No & $2.4(3 / 125)$ & $7.1(5 / 70)$ \\
\hline \multicolumn{3}{|c|}{ 6: Would you refuse the same colon cleansing medication again if it were to be prescribed to you in the future? } \\
\hline Yes & $3.2(4 / 125)$ & $5.7(4 / 70)$ \\
\hline No & $96.8(121 / 125)$ & $94.3(66 / 70)$ \\
\hline
\end{tabular}

\section{Repeat colonoscopy}

A statistically significant difference in the proportion of patients requiring a repeat colonoscopy due to poor colon preparation was observed based on a post-hoc analysis using Fisher's exact test $(P<0.0001)$. A total of three $(2.4 \%)$ patients following the TD regimen and $15(21.4 \%)$ patients following the DBD regimen required a repeat colonoscopy due to poor colon preparation.

\section{Safety}

Overall incidence of treatment-emergent AEs was 17 events (in 15 patients: $12.0 \%$ ) in TD regimen and seven events (in four patients: $5.7 \%$ ) in DBD regimen. The difference in the incidence of treatment-emergent AEs between the 2 regimens was not observed to be statistically significant, based on a post-hoc analysis using Fisher's exact test $(P=0.2988)$. The most common AEs were gastrointestinal disorders (7.2\%), with abdominal pain being the most common AE (TD 5 [4\%] patients, and none in DBD) ( $>$ Table 5). Large intestinal obstruction and post-procedural haemorrhage were considered as SAEs observed with TD,
- Table 5 Most frequent adverse events (at least $1 \%$ in any treatment group) by preferred term.

\begin{tabular}{|l|l|l|}
\hline & $\begin{array}{l}\text { Tailored dosing } \\
(\mathbf{N}=125)\end{array}$ & $\begin{array}{l}\text { Day-before dosing } \\
(\mathbf{N}=\mathbf{7 0})\end{array}$ \\
\hline Adverse events, n (\%) & $15(12.0)$ & $4(5.7)$ \\
\hline - Gilbert's syndrome & - & $1(1.4)$ \\
\hline - Abdominal pain & $5(4.0)$ & - \\
\hline - Diarrhea & $1(0.8)$ & $1(1.4)$ \\
\hline - Nausea & $1(0.8)$ & $1(1.4)$ \\
\hline - Eructation & - & $1(1.4)$ \\
\hline - Malaise & - & $1(1.4)$ \\
\hline - Hypercalcaemia & - & $1(1.4)$ \\
\hline \% & & $1(1.4)$ \\
\hline N, number of patients; $n$ number of patients with adverse events;
\end{tabular}


both unrelated to PICOPREP. No clinically relevant findings were observed in haematology, clinical chemistry, and coagulation laboratory parameters. A transient, average increase from baseline in serum magnesium (10.5\%) and bilirubin (49.2\%) was observed on the day of colonoscopy which was similar across both treatment regimens and well within the limits of normal range. No deaths were reported in the study.

\section{Discussion}

This study demonstrated that the TD regimen was superior to the DBD regimen in overall and ascending colon cleansing efficacy. For mid (transverse and descending) and recto-sigmoid colon cleansing, the odds that the Ottawa Scale score was $\leq 1$, were statistically significantly greater for the TD regimen compared to the DBD regimen. The ease of consuming and overall experience were rated as easy to very easy and good to excellent, respectively, and were comparable for both dosing regimens. Furthermore, the incidence of repeat colonoscopy was significantly lower in the TD regimen (3 patients) as compared with the DBD regimen (15 patients).

The TD regimen demonstrated statistically significant improved colon cleansing for all colon segments (ascending, mid and recto-sigmoid colon) compared with the DBD regimen. This benefit can be attributed to the recommended short time period between the last dose of bowel preparation and colonoscopy procedure due to customised timing of the second dose (6.06 and 19.74 hours for TD and DBD regimen, respectively) $[8,26]$.

The TD regimen had a reduced impact on ability to perform daily non-work-related activities in comparison to the DBD regimen. These results are in line with previous SEE CLEAR study with similar acceptability and tolerability profile of PICOPREP $[13,17]$.

Of the 195 patients exposed to study drug, 19 experienced a treatment emergent $\mathrm{AE}$. The most common AEs belonged to the category Gastrointestinal Disorders by System Organ Class (preferred term, abdominal pain). The safety results of this study were in line with the real-life experiences $[6,26]$ and further support the well-established safety and tolerability profile of PICOPREP irrespective of the dosing regimens.

The current study has several strengths. First, randomization was stratified by the time of colonoscopy to ensure balance between the treatment arms with respect to the number of morning and afternoon colonoscopies. The analyses were also adjusted for time of colonoscopy, to account for any potential imbalance between the treatment arms, occurring by chance due to the randomisation. Furthermore, to minimize evaluation bias, the colonoscopist assessing the outcome of the colon cleansing was blinded to the dosing schedules.

Importantly, the efficacy analyses in ITT and PP populations were given equal weight to evaluate the non-inferiority of TD regimen with the DBD regimen. This is in line with recommendations from regulatory bodies as the analysis based on the ITT populations is not necessarily considered conservative for a non-inferiority study as it would be for a superiority study.

\section{Conclusion}

The TD regimen was superior compared with the DBD regimen in ascending and overall colon cleansing in preparation for colonoscopy. PICOPREP was safe and tolerable in both dosing regimens. The TD regimen is therefore efficacious in bowel cleansing prior to colonoscopy, regardless of the planned time of colonoscopy.

\section{Acknowledgements}

The authors take full responsibility for the content of this manuscript. This study was sponsored by Ferring Pharmaceuticals. Medical writing support was provided by Mohit Joshi and Dr. Payal Bhardwaj (Tata Consultancy Services), both funded by Ferring. The authors also thank the following people from Ferring Pharmaceuticals for their contribution to the OPTIMA study: Christine Angelin, Elda Dinaj, Marianne Vinther Ammitzbøll, Johan Masure, Kyle Raymond, Winston Man A Hing.

\section{Competing interests}

Ralf Kiesslich has received research support and speaker fee from Ferring pharmaceuticals. Stefan Schubert has received grants from Ferring pharmaceuticals for travel and meetings for this study. Michael Klemt-Kropp has received grants from Gilead Siences for an observational study "Retrieval of patients chronically infected with Hep. B and C in Northern Holland". He has also received fees from AbbVie as a consulting member of international advisory Board hepatitis C. Furthermore, he has received fees for lectures, presentations and for moderating medical events on hepatitis B and C. Imke Behnken has received payments for lectures including service on speaker's bureaus from MSD, Astra Zeneca and Novartis. Gillaume Bonnaud has received consulting fees/honarium from Ferring pharmaceuticals, Abbvie, Aptacis, MSD, Covidien and Takeda. He has also received travel allowance for the meetings and manuscript preparation and fees for participation in review activities such as data monitoring boards. Michael Blaker has received payments for lectures and chairs at symposia and conventions from Ferring Pharmaceuticals. Thierry Ponchon has received grants from Boston Scientific, Cook and Olympus Co. He has received consulting fees or honorium from Olympus Co, Boston Scientific, Cook Medical and Mayloy Spindler. He has also received fees for participation in review activities from Norgine and payments for lectures including service on speaker bureaus from Olympus Co, Boston Scientific, Cook Medical Fujifilm and Covidien.

Michael Mross, Tobias Klugmann, Eric Keulen, Marcel Groenen and Wilfred Landry have no conflict of interests to disclose. Meredin Stoltenberg is an employee of Ferring pharmaceuticals. 


\section{References}

[1] Jemal A, Bray F, Center MM et al. Global cancer statistics. CA Cancer J Clin 2011; 612: 69-90

[2] Prakash SR, Verma S, McGowan J et al. Improving the quality of colonoscopy bowel preparation using an educational video. Can ] Gastroenterol 2013; 27: $696-700$

[3] Hagege H, Laugier R, Nahon S et al. Real-life conditions of use of sodium phosphate tablets for colon cleansing before colonoscopy. Endosc Int Open 2015; 3: 346-353

[4] Shah H, Desai D, Samant $\mathrm{H}$ et al. Comparison of split-dosing vs nonsplit (morning) dosing regimen for assessment of quality of bowel preparation for colonoscopy. World J Gastrointest Endosc 2014; 6: $606-611$

[5] Yoo IK, Lee JS, Chun HJ et al. A randomized, prospective trial on effi cacy and tolerability of low-volume bowel preparation methods for colonoscopy. Dig Liver Dis 2015; 47: 131 - 137

[6] Jeon SR, Kim HG, Lee JS et al. Randomized controlled trial of low-volume bowel preparation agents for colonic bowel preparation: $2-\mathrm{L}$ polyethylene glycol with ascorbic acid versus sodium picosulfate with magnesium citrate. Int ] Colorectal Dis 2015; 30: $251-258$

[7] Hassan C, Bretthauer MF, Kaminski MF et al. Bowel preparation for colonoscopy: European Society of Gastrointestinal Endoscopy (ESGE) guidelines. Endoscopy 2013; 45: 142 - 150

[8] Mathus-Vliegen E, Pellise M, Heresbach D et al. Consensus guidelines for the use of bowel preparation prior to colonic diagnostic procedures: colonoscopy and small bowel video capsule endoscopy. Curr Med Res Opin 2013; 29: 931 - 945

[9] Johnson D, Barkun A, Cohen L et al. Optimizing adequacy of bowel cleansing for colonoscopy: recommendations from the US multi-society task force on colorectal cancer. Gastroenterology 2014; 147: $903-924$

[10] Rex D. Optimal bowel preparation - a practical guide for clinicians. Nat Rev Gastroenterol Hepatol 2014; 11: 419-425

[11] Rex D. Bowel preparation for colonoscopy: entering an era of increased expectations for efficacy. Clin Gastroenterol Hepatol 2014; 12: $458-462$

[12] Oh CH, Lee CK, Kim JW et al. Suboptimal bowel preparation significantly impairs colonoscopic detection of non-polypoid colorectal neoplasms. Dig Dis Sci 2015; 60: 2294 - 2303

[13] Rex DK, Katz PO, Bertiger G et al. Split-dose administration of a dualaction, low-volume bowel cleanser for colonoscopy: the SEE CLEAR I study. Gastrointest Endosc 2013; 78: 132 - 141
[14] Manes G, Repici A, Hassan C ;MAGIC-P study group Randomized controlled trial comparing efficacy and acceptability of split- and standard-dose sodium picosulfate plus magnesium citrate for bowel cleansing prior to colonoscopy. Endoscopy 2014; 46: $662-669$

[15] Kim ES, Lee WJ, Jeen YT et al. A randomized, endoscopist-blinded, prospective trial to compare the preference and efficacy of four bowel-cleansing regimens for colonoscopy. Scand J Gastroenterol 2014; 49: $871-877$

[16] Song KH, Suh WS, Jeong JS et al. Effectiveness of sodium picosulfate/ magnesium citrate (PICO) for colonoscopy preparation. Ann Coloproctol 2014; 30: $222-227$

[17] Katz PO, Rex DK, Epstein M et al. A dual-action, low-volume bowel cleanser administered the day before colonoscopy: results from the SEE CLEAR II study. Am J Gastroenterol 2013; 108: 401 -409

[18] Muñoz-Navas M, Calleja JL, Payeras G et al. A randomized trial to compare the efficacy and tolerability of sodium picosulfate-magnesium citrate solution vs. $4 \mathrm{~L}$ polyethylene glycol solution as a bowel preparation for colonoscopy. J Colorectal Dis 2015; 30: 1407 - 1416

[19] Bertiger G, Jones E, Dahdal DN et al. Serum magnesium concentrations in patients receiving sodium picosulfate and magnesium citrate bowel preparation: an assessment of renal function and electrocardiographic conduction. Clin Exp Gastroenterol 2015; 28: 215 - 224

[20] Leitao K, Grimstad T, Bretthauer M et al. Polyethylene glycol vs sodium picosulfate/magnesium citrate for colonoscopy preparation. Endosc Int Open 2014; 2: E230 - E234

[21] Kim HG, Huh KC, Koo HS et al. Sodium picosulfate with magnesium citrate (SPMC) plus laxative is a good alternative to conventional large volume polyethylene glycol in bowel preparation: a multicenter randomized single-blinded trial. Gut Liver 2015; 9: 494 - 501

[22] Turner D, Benchimol El, Dunn H et al. Pico-Salax versus polyethylene glycol for bowel cleanout before colonoscopy in children: a randomized controlled trial. Endoscopy 2009; 41: 1038-1045

[23] Di Nardo G, Aloi M, Cucchiara S et al. Bowel preparations for colonoscopy: an RCT. Pediatrics 2014; 134: 249-256

[24] Vejzovic V, Wennick A, Idvall E et al. Polyethylene glycol or sodium picosulphate based laxatives before colonoscopy in children. J Pediatr Gastroenterol Nutr 2016; 62: 414-419

[25] Hahn S. Understanding noninferiority trials. Korean J Pediatr 2012; 55: $403-407$

[26] Rex DK, Imperiale TF, Latinovich DR et al. Impact of bowel preparation on efficiency and cost of colonoscopy. Am J Gastroenterol 2002; 97 : $1696-1700$ 


\section{Reactions to PICOPREP used in the study}

1. How easy or difficult was it to consume the PICOPREP medication? (please circle one)

$\begin{array}{ccccc}1 & 2 & 3 & 4 & 5 \\ \text { Very Easy } & \text { Easy } & \text { Tolerable } & \text { Difficult } & \text { Very Difficult }\end{array}$

2. Were you able to consume the entire PICOPREP medication as instructed?

Yes No

3. Please describe your overall experience with the PICOPREP medication.

Please circle one of these five terms:

$\begin{array}{ccccc}1 & 2 & 3 & 4 & 5 \\ \text { Excellent } & \text { Good } & \text { Fair } & \text { Poor } & \text { Bad }\end{array}$

4. The taste of the PICOPREP medication was: (please circle one)

$\begin{array}{ccccc}1 & 2 & 3 & 4 & 5 \\ \text { Excellent } & \text { Good } & \text { Fair } & \text { Poor } & \text { Bad }\end{array}$

5. Would you ask your doctor for this colon cleansing medication again if you need another colonoscopy in the future?

Yes No

6. Would you refuse the same colon cleansing medication again if it were to be prescribed to you in the future?

Yes No

\section{Experience with previous colon cleansing treatment taken in the recent past}

7. Have you had any colonoscopy before (within the past 3 years)?

Yes No

If yes, please complete questions below

7a. If YES, then please provide the type of colon cleansing medication you used in your most recent colonoscopy:
2L prep
$4 \mathrm{~L}$ prep
Tablet prep
Over-the-counter-laxatives Other
Unknown

7b. If YES, then please provide the name of the colon cleansing medication you used in your most recent colonoscopy:

Name:

7c. If YES, were you able to complete as instructed the entire colon cleansing medication you used in your most recent colonoscopy?

Yes No Don't remember

7d. If YES, would you describe the colon cleansing medications you received for this colonoscopy as: (please circle one)

\section{1}

Much Easier

to Complete
2

Easier

to Complete
3

The Same
4

Harder

to Complete
5

Much Harder

to Complete 